\title{
Transmission Electron Tomography of Adeno-Associated Virus (AAV)
}

\author{
G. Gao and G. M. Hendricks* \\ University of Massachusetts Medical School \\ Gene Therapy Center, Cell Biology*, Worcester, Massachusetts 01655
}

Adeno-associated virus (AAV) is a small (25-nm), nonenveloped virus that packages a linear singlestranded DNA genome. It belongs to the family Parvoviridae and is placed in the genus Dependovirus. Productive infection by AAV occurs only in the presence of a helper virus, either adenovirus or herpesvirus. Twelve human serotypes of AAV (AAV serotype 1 [AAV-1] to AAV12) and more than 100 natural AAV variants from nonhuman primates have been discovered to date [1]. The lack of pathogenicity of the virus, it's persistence in the host and the many available serotypes are what makes AAV's such a good potential delivery vehicle for gene therapy applications.

The use of AAV vectors for gene expression can be advantageous when immunogenicity is a concern. A crude or contaminated AAV preparation may contain material that negates this benefit. It is therefore critical that the virus be highly pure to minimize an immunogenic response in the host cell [2]. Ultimately, to assure the purity of the AAV prep electron microscopy analysis is necessary. Negative stain EM analysis is used to shows the viral capsid preps are pure and whether they are full vs. empty. However, the question of what is full vs. empty has only been supposed based on how negative staining works to fill in the negative outline and fine structure of the samples being examined.

In this study we take up this question and apply the latest electron microscopy tomographic techniques combined with negative staining to demonstrate the conformational difference between viral capsids that have been filled and those that remain empty. Ultimately, we hope that this structural information on the AAV virus capsid will provide a three-dimensional platform for the further investigations to improve specific cell/tissue-targeting gene-delivery applications for these viral vectors.

References:

1. O. Quesada, B. Gurda, L. Govindasamy, R. McKenna, E. Kohlbrenner, G. Aslanidi, S. Zolotukhin, N. Muzyczkac and M. Agbandje-McKennaa (2007)*Production, purification and preliminary X-ray crystallographic studies of adeno-associated virus serotype 7. Acta Cryst. F63, 1073-1076.

2. S. Daya and K. I. Berns (2008) Gene Therapy Using Adeno-Associated Virus Vectors. CLINICAL MICROBIOLOGY REVIEWS, Oct., p. 583-593. 

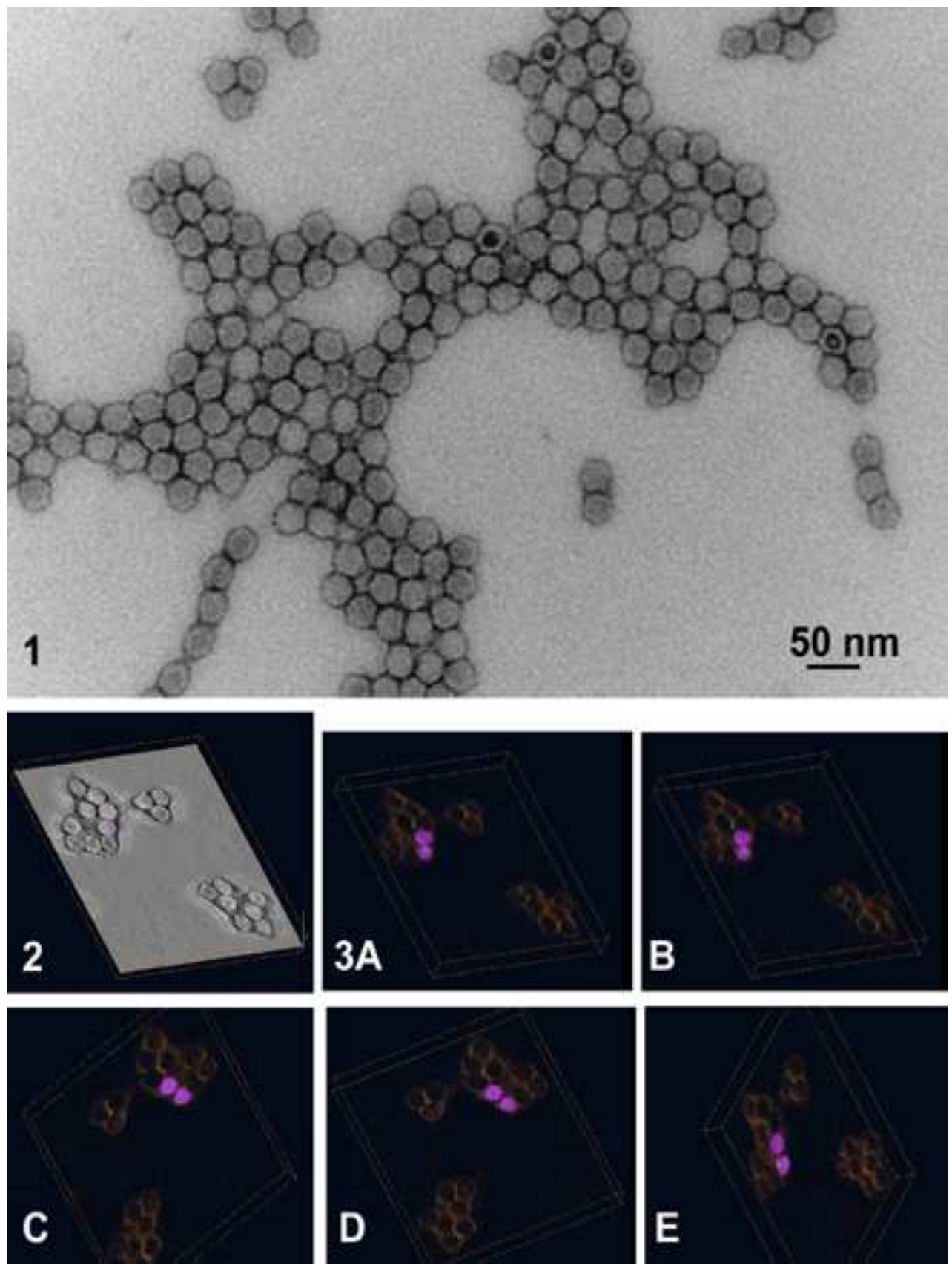

Figure 1. Negative stained AAV particles. Spread onto a carbon stabilized Formvar support film and stained with uranyl acetate.

Figure 2. Tomagraphic reconstruction data set.

Figure 3A, B, C, D, and E. Tomagraphic rotational series. The empty AAV particle is actually dimpled on both the top and bottom. 